\title{
Emission Spectroscopic Measurements with an Optical Probe in the NASA Ames IHF Arc Jet Facility
}

\author{
Michael W. Winter ${ }^{1}$ \\ University Affiliated Research Center, UC Santa Cruz, NASA Ames Research Center, Moffett Field, CA 94035 \\ Dinesh K. Prabhu ${ }^{2}$ \\ ERC, Incorporated, NASA Ames Research Center, Moffett Field, CA 94035 \\ and
}

George A. Raiche, ${ }^{3}$ Imelda Terrazas-Salinas, ${ }^{4}$ Frank C. L. Hui ${ }^{5}$

NASA Ames Research Center, Moffett Field, CA 94035

\begin{abstract}
An optical probe was designed to measure radiation (from inside the arc heater) incident on a test sample immersed in the arc-heated stream. Currently, only crude estimates are available for this incident radiation. Unlike efforts of the past, where the probe line of sight was inclined to the nozzle centerline, the present development focuses on having the probe line of sight coincide with the nozzle centerline. A fiber-coupled spectrometer was used to measure the spectral distribution of incident radiation in the wavelength range of 225 to $900 \mathrm{~nm}$. The radiation heat flux in this wavelength range was determined by integration of measured emission spectral intensity calibrated to incident irradiance from an integrating sphere. Two arc-heater conditions, corresponding to stream bulk enthalpy levels of 12 and $22 \mathrm{MJ} / \mathrm{kg}$, were investigated in the 13-inch diameter nozzle of the Interaction Heating Facility at NASA Ames Research Center. With the probe placed at a distance of 10 inches from the nozzle exit plane, total radiative heat fluxes were measured to be 3.3 and $8.4 \mathrm{~W} / \mathrm{cm}^{2}$ for the 12 and $22 \mathrm{MJ} / \mathrm{kg}$ conditions, respectively. About $\mathbf{1 7 \%}$ of these radiative fluxes were due to bound-bound radiation from atoms and molecules, while the remaining $83 \%$ could be attributed to continua (bound-free and/or free-free). A comparison with spectral simulation based on CFD solutions for the arc-heater flow field and with spectroscopic measurements in the plenum region indicates that more than $95 \%$ of the measured radiation is generated in the arc region. The total radiative heat flux from the line radiation could increase by a factor of two through contributions from wavelengths outside the measured range, i.e., from the vacuum ultraviolet (wavelengths less than $225 \mathrm{~nm}$ ) and the infrared (wavelengths greater than $900 \mathrm{~nm}$ ). An extrapolation of the continuum radiation to these two wavelength regions was not attempted. In the tested configuration, the measured radiative heat flux accounts for only about $1.4 \%$ of the nominal heat flux on a flat face model and therefore is considered negligible. In the 6 -inch diameter nozzle, on account of shorter path lengths, the radiation heat flux could be significant. Therefore, future tests in the 6-inch nozzle will have radiometers in addition to the optical probe.
\end{abstract}

\section{Introduction}

$\mathrm{T}$ HE development and qualification of heatshield materials for high-speed atmospheric entries relies heavily on ground testing in high enthalpy plasma facilities such as the arc jets at NASA Ames Research Center (ARC). One of the key parameters for material testing is the heat flux to a material sample. Calorimeter measurements are used to determine the heat flux characteristic for the chosen test conditions. So far, the heat flux to a surface is assumed to be solely determined by the properties of the plasma forming behind the bow shock in front of the material sample. A high-temperature plasma, however, emits a significant amount of

\footnotetext{
${ }^{1}$ Research Scientist, Aerothermodynamics Branch, M/S 230-3, Senior Member AIAA.

${ }^{2}$ Senior Research Scientist, Aerothermodynamics Branch, MS 230-3. Associate Fellow AIAA

${ }^{3}$ Branch Chief Thermo-Physics Facilities Branch, MS 229-2. Member AIAA

${ }^{4}$ Lead Test Engineer, Thermo-Physics Facilities Branch, MS 229-4

${ }^{5}$ Test Engineer, Thermo-Physics Facilities Branch, MS 229-4. Member AIAA
} 
radiation and constricted arc heaters produce an extended plasma volume at high temperature and pressure inside the heater. The plasma radiation will hit the surface of a probe or a material sample in the plasma beam and contribute to the heating. The effect of radiative heating will be different on a metallic heat flux probe and a material sample due to the different absorption coefficients, therefore biasing the heat flux measurements. In consequence, the material sample will typically "see" a higher heat flux than the metallic calorimeter. The goal of this work is to measure this radiative heating and quantify its contribution during arc-jet testing.

The $60 \mathrm{MW}$ Interaction Heating Facility (IHF), one of several arc jet facilities at NASA Ames Research Center, is used to test and qualify spacecraft thermal protection materials in "flight-like" aerothermal environments. The IHF heater is a constricted arc design with an upstream anode, and a downstream cathode, which consists of 8 electrode disks. The working gas is injected along the arc-column. The discharge is established between the anode and the cathode, and the working gas is heated to temperatures of $8000 \mathrm{~K}$ or more. The magnitude of these temperatures, hence the flow energy, depend on the input current, voltage, and flow rates. Following the cathode, there are 4 disks/segments through which additional room temperature air can be injected to tailor the enthalpy of the working medium to the desired test value. This set of 4 segments is referred to as the "plenum" in the present work. The working gas, of appropriate enthalpy, is then expanded into the test chamber through a convergent-divergent nozzle, and the test article is placed in the free jet. Different nozzle geometries, mainly defined by the nozzle exit diameter, can be used to obtain various heat fluxes and pressures on a material sample. For this work, nozzle exit diameters of 6" and 13" are used. Figure 1 shows a sketch of the IHF design.

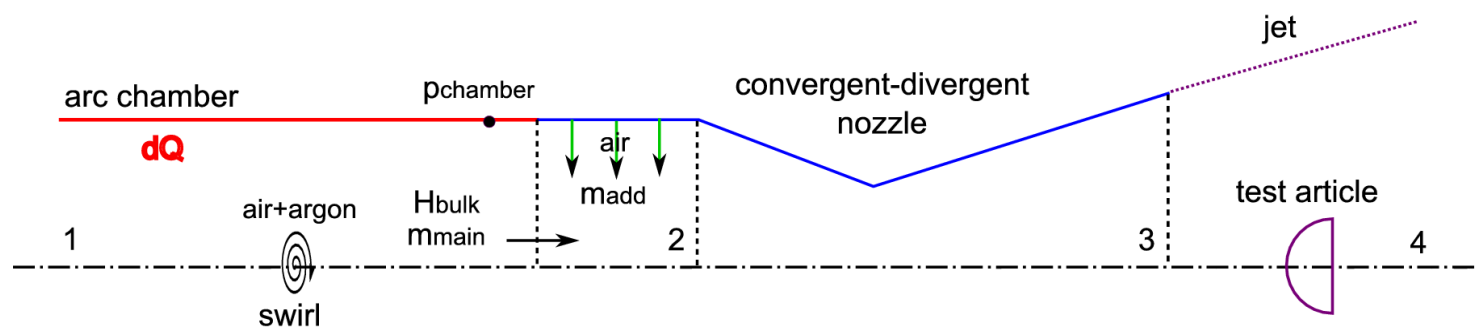

Figure 1. Sketch of the IHF working principle.

Over the last two years measurement campaigns involving optical emission spectroscopy for the characterization of re-entry plasmas in the Ames arc-jet facilities have been conducted. These measurement campaigns focused on the plenum ${ }^{1}$ of the IHF arc-heater and the free jet ${ }^{2}$ in the test chamber to support facility characterization and provide validation data for CFD simulations. Both equilibrium conditions in the plenum and non-equilibrium effects in the free stream were discussed in the work of Winter et al. ${ }^{1,2}$

Recently, an optical probe was designed to measure incident radiation on a sample in the arc-jet flow to quantify the radiative loads by measurements. Prior to this work, there were only estimates available for the radiation from the inside of the heater impinging on a material sample or probe surface. In a first attempt, the impinging radiation was measured spectrally resolved with a fiber coupled spectrometer.

\section{Measurement Strategy and Instrumentation}

The test strategy adopted was to sweep the optical probe through the free jet (Fig. 2) with a defined sweep velocity. During that time the spectrometer continuously acquired data and stored them in the local (on-board) memory. Maximum data acquisition speeds are on the order of $250 \mathrm{~Hz}$ with minimum acquisition times of $1.1 \mu \mathrm{s}$ for each individual spectrum. The data acquisition of the spectrometer was triggered by a $5 \mathrm{~V}$ TTL (TransistorTransistor Logic) pulse once the probe was set into motion. Both trigger and position readings of the sting arm were stored in the facility data acquisition system to enable synchronization with the spectra stored in the internal memory of the spectrometer.

During the sweep, the probe entered the flow and measured post shock radiation while still looking at the cold nozzle wall. The IHF arc-jet flow shows a rather flat profile so the emission during that time can be considered to be close to the post shock emission in the center line region, at least with the spatially resolving setup. Free stream emission is truly negligible in comparison to the post-shock emission. The measured data showed that, in comparison to the emission coming from inside the arc heater, the post shock emission was negligible as well. Close to the centerline, the probe started seeing the superposition of nozzle throat, plenum, and arc-channel emission. Figure 2 illustrates the measurement strategy showing three probe positions during the sweep with the probe outside the flow, looking at the nozzle wall with a shock already building up and at the center line measuring the sum of plenum and post-shock emission.

The tests were conducted at known arc heater conditions with existing numerical solutions of the flow field obtained with the computational fluid dynamics (CFD) code DPLR. ${ }^{3,5}$ 
The optical signals were collected through a combination of two optical fibers of $2.5 \mathrm{~m}$ length which were routed to an optical table outside the test chamber through a vacuum duct and connected to an Ocean Optics filter coupler. Neutral density

optical probe

b)

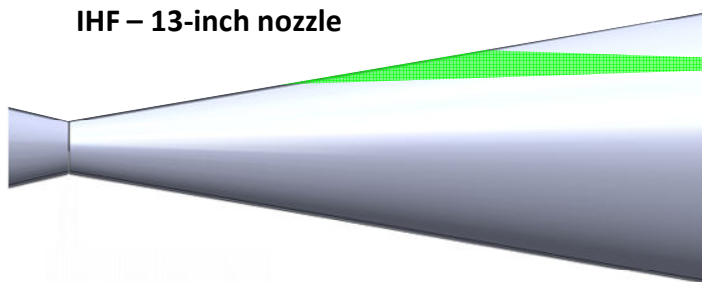

optical probe

c)

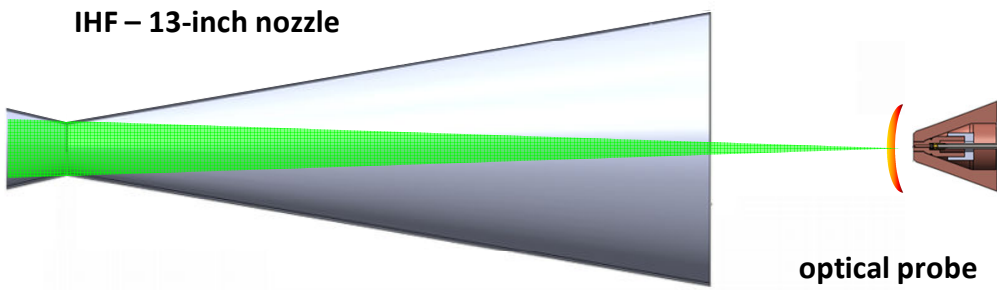

Figure 2. Measurement strategy during Optical Probe sweep through the plasma flow of the IHF arc-jet. a) Optical probe outside the plasma flow, b) probe "seeing' the cold wall, and c) at the center line.

filters were used to further reduce the signal strength. The filter holder was coupled through one more fiber of $1 \mathrm{~m}$ length to the spectrometer (Avantes AvaSpec-3648USB2-UA, nominal wavelength range $180-1100 \mathrm{~nm}$ ). In the used configuration, the useful wavelength range was 22-900nm with a free width at half maximum (FWHM) of about $1.3 \mathrm{~nm}$. Acquisition times during arc-jet testing varied between $10 \mu \mathrm{s}$ and $400 \mu \mathrm{s}$.

Data analysis involves spectral simulation with the line-by-line radiation code NEQAIR ${ }^{6}$ using the numerical flow field data as input.

In parallel to the optical probe measurements, plenum spectroscopy similar to prior measurement campaigns ${ }^{1}$ was conducted to obtain additional information about the plasma condition prior to its expansion in the nozzle.The optical measurements were accompanied by standard flow characterization techniques used at Ames in the form of stream survey sweeps for measurements of pressure and heat flux distribution across the test stream, and single-point center line heat flux measurements.
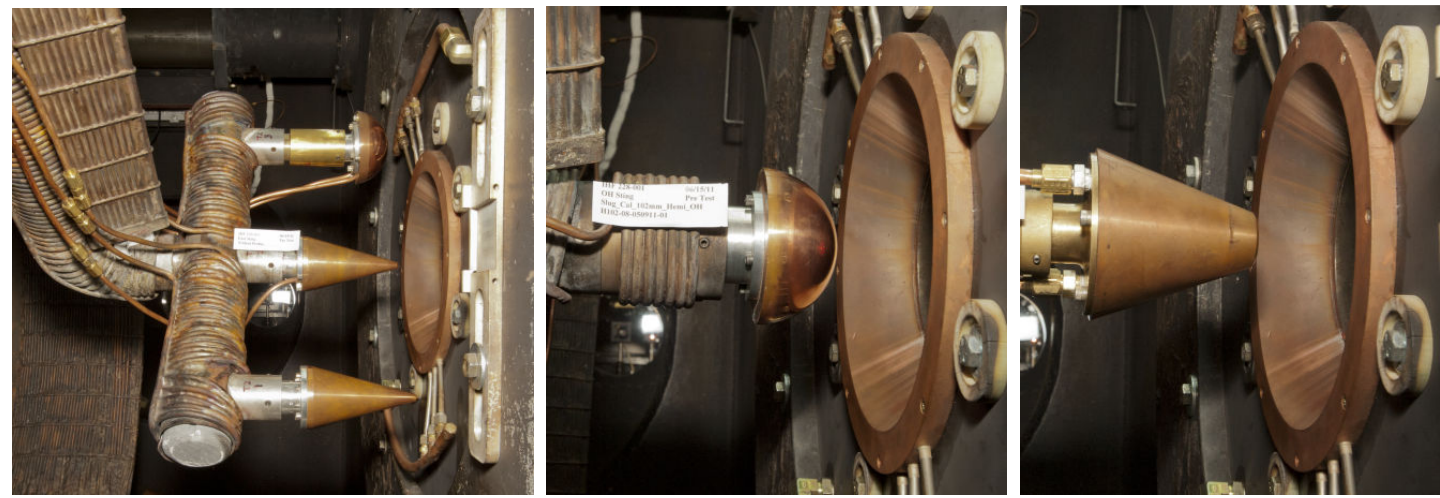

Figure 3. Trident probe, Slug calorimeter, and optical probe mounted on the IHF sting arms during testing with the IHF 6 inch nozzle.

For each condition, centerline heat flux and pressure were measured with a 102-mm-diameter hemispherical slug calorimeter, and sweeps through the plasma beam were performed with a trident probe at a sweep speed of 60 inches $/ \mathrm{s}$ (about $1.5 \mathrm{~m} / \mathrm{s}$ ). The trident probe was configured with a $102-\mathrm{mm}$-diameter hemispherical probe with a null point calorimeter and a pressure port, a $4.57-\mathrm{mm}$ - nose-radius sphere/cone probe with a null point calorimeter, and a 4.57-mm-nose-radius sphere cone probe with a pressure port. Figure 3 shows photos of all probes mounted on the IHF sting arms at the nozzle center line. 


\section{Radiation Transfer}

To transform the known radiation from a calibration lamp calibrated to spectral radiance in $\mathrm{W} /\left(\mathrm{cm}^{2} \mathrm{sr} \mathrm{nm}\right)$ or calculated plasma radiation (usually given in radiance values, too) into a incident irradiance on the optical probe in $\mathrm{W} / \mathrm{cm}^{2}$ (equivalent to radiation heat flux), simple geometric considerations can be made.

On the optical axis, the heat flux $Q$ to a surface $A$ emitted by a point source emitting the radiant flux $P$ (in $\mathrm{W} / \mathrm{sr}$ ) in a distance $x_{0}$ to the surface is given by

$$
Q=P \frac{\Omega}{A}
$$

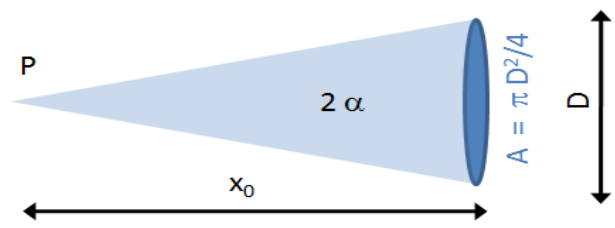

Figure 4. Geometry for a point source on the optical axis in a distance $x_{0}$ to a surface $A$.

with the receiving surface $\quad A=\pi \frac{D^{2}}{4}$

and the solid angle

$$
\Omega=2 \pi(1-\cos (\alpha))=2 \pi\left(1-\cos \left(\arctan \left(\frac{D}{2 x_{0}}\right)\right)\right)
$$

For small angles $\left(\tan (\alpha)=\alpha, \quad \cos (\alpha)=1-\alpha^{2} / 2\right), \Omega$ becomes

$$
\Omega=\pi \frac{D^{2}}{\left(4 x_{0}\right)^{2}}
$$

and the heat flux is given by

$$
Q=\frac{P}{x_{0}^{2}}
$$

If the line of sight is inclined at an angle $\theta$ to the surface $A$, the solid angle to a circular surface element perpendicular to the optical axis becomes

$\Omega_{\perp}=2 \pi\left(1-\cos \left(\arctan \left(\frac{D \cos (\theta)}{2 x_{0}}\right)\right)\right)$

The receiving surface, needed for the calculation of the heat flux, stays the same as before, but the projected area, needed for the solid angle, now is an ellipse. If the elliptic solid angle is approximated by

It can be written as

$$
\Omega(\theta)=\Omega_{\perp} \frac{A_{\text {ellipse }}}{A_{\text {circle }}}=\Omega_{\perp} \cos (\theta)
$$

Figure 5. Geometry for a point source on an optical axis unde an angle $\theta$.

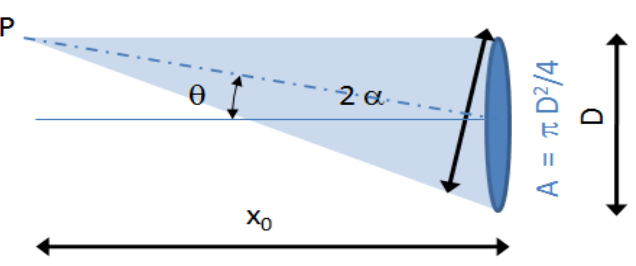

$$
\Omega(\theta)=\frac{\pi D^{2} \cos (\theta)^{3}}{2 x_{0}^{2}}
$$

and the heat flux becomes

$$
Q=P \frac{\Omega}{A}=P \frac{\cos (\theta)^{3}}{x_{0}^{2}}
$$

This means, the sensitivity of a point in the field of view of the optical probe decreases with the third power of $\cos (\theta)$ as plotted in Fig. 6. As will be shown later, the maximum angles for the measurements described here are below $2^{\circ}$. Therefore, equation (5) can be used as a very good approximation and the sensitivity of the probe is considered constant across the field of view. Obviously, all considerations above are valid for the spectral quantities (spectral radiance and irradiance) as well.

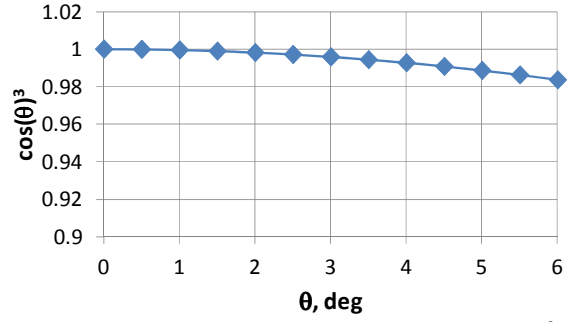

Figure 6. Contribution of the $\cos (\theta)^{3}$ term to the sensitivity in the field of view 


\section{Probe Design and Alignment}

The probe design (Fig. 7) is based on a water-cooled probe body that carries an optical plug with a sapphire window to seal the optical path against the post shock plasma and a mechanical connection of an optical fiber. The first design, which included a Quartz glass collimator to enable alignment of the probe using a laser, allowed for spatial resolution of the nozzle throat section with 6 independent measurements. The next design eliminated the collimator by using limiting apertures in the optical path to provide a sufficient field of view to cover all radiation emitted from inside the arc heater. On the arc-jet side, the limiting aperture is the nozzle throat, which defines the necessary field of view. On the probe side, the front mask is the limiting aperture stop. The spectrometer was located outside the arc-jet test box and the optical signals were fed to the spectrometer by optical fibers.

The design makes use of heritage from the plenum spectroscopy development in using the optical plugs that carry a sapphire window, two spacers, an O-ring, and an insert to apply a sealing force in a threaded housing. ${ }^{1}$ This unit can be mounted in the optical path as an entity. This design has been proved to work under the harsh environment of the arc-jet plenum chamber. ${ }^{1}$ A collimator was included to enable probe alignment by backilluminating the fiber with a laser. Without the collimator, the laser exits the fiber as a strongly diverging beam and is no longer visible in the IHF nozzle throat which was used as alignment target. With the collimator, the measurement spot, as inferred from the image of the laser going backwards through the system, had a size of about $10 \mathrm{~mm}$ at a distance of $362 \mathrm{~mm}$ (14.25 inches) corresponding to the distance to the nozzle throat during that test.

The rear plug and the front plug are threaded into the water-cooled probe body. The rear plug is mounted from the back of the probe before mounting it on the sting arm and carries the aforementioned optics and the collimator that connects the optical path to the optical fiber. The front plug can be inserted and taken out while the probe is mounted and aligned. Therefore, different entrance apertures can be used without changing the alignment.

One major result from the tests with the spatially resolving design was that the shock/post-shock radiation does not significantly contribute to the radiation heat flux and can be neglected compared to the radiation from the inside of the arc-heater. During the first tests, it turned out that, even with the laser alignment, the alignment accuracy was not sufficient, resulting in non-symmetric radiation profiles during the sweep. In addition, determining an integrated value of irradiance to the probe surface was complicated through the diverging field of view. With distance, the measured spots overlap more and more and the integration requires knowledge of the axial intensity distribution. To ensure thorough results, the spatial resolution had to be either increased until all relevant cross sections in the optical path are independent from each other for single measurements during the sweep, or decreased until the center line measurement includes the whole radiating volume inside the arc heater. In conclusion, the use of the collimator did not yield the desired results. To improve the design, the fiber was illuminated without focusing elements and limiting the optical path through aperture stops was implemented. Figure 7 shows the second generation design. This, however, required a different alignment strategy without a laser back-illuminating the fiber.
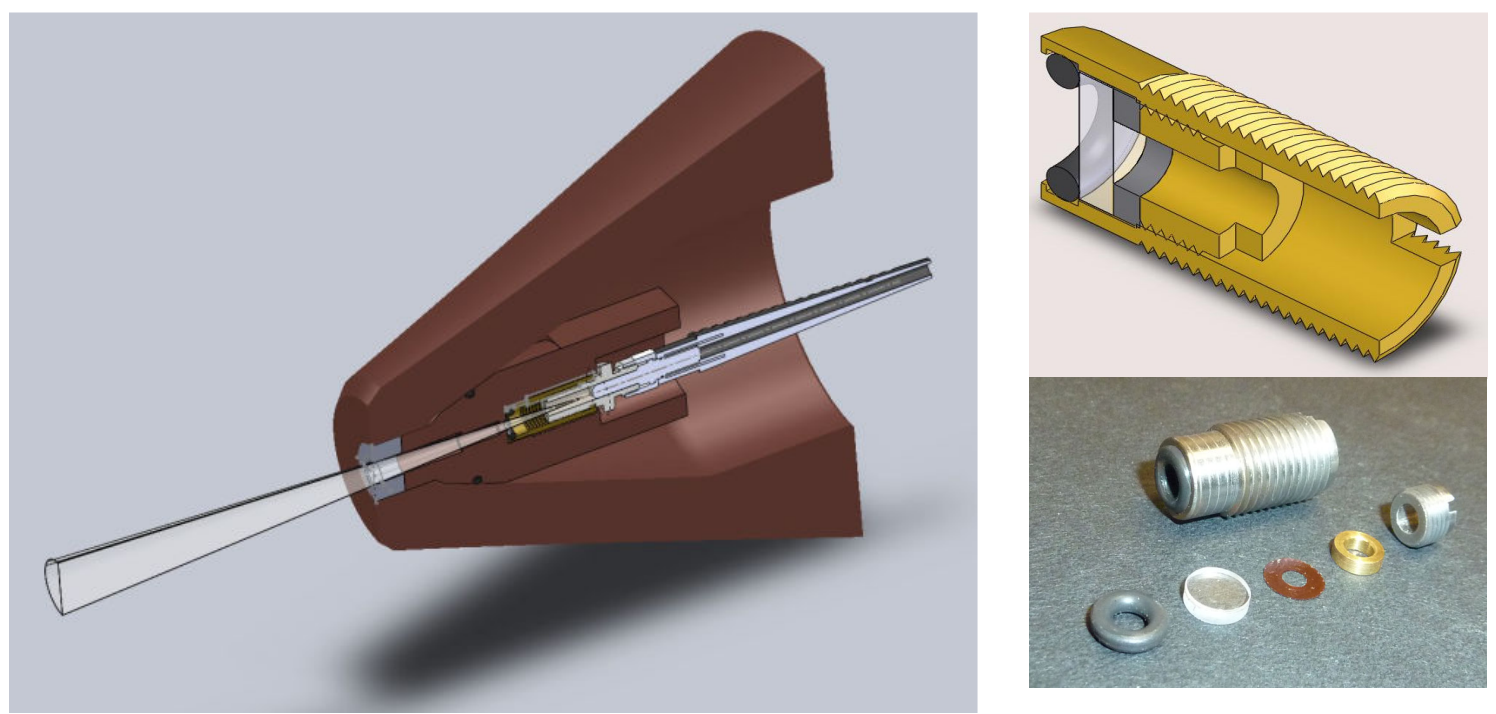

Figure 7. Second generation design without focusing element. 
The optical path is limited through aperture stops only, without focusing elements. Thus, each point in the field of view sees, in good approximation, the same receiving surface (the fiber aperture) which corresponds to very similar solid angles in which radiation is emitted. The sensitivity is almost constant across the field of view. However, the coupling into the fiber under different angles might cause slight variations in sensitivity. The necessary field of view for testing in the IHF equipped with the 13" nozzle is defined by the nozzle throat diameter which is the limiting aperture stop for radiation coming from the inside of the heater. At a probe position of 10 inches ) from the nozzle exit, the half-angle of the necessary field of view becomes $1.9^{\circ}$ which is also the maximum angle under which radiation has to be fed into the fiber. This value is clearly smaller than the critical acceptance angle of the optical fiber of $\pm 12.7^{\circ}$, as defined by its numerical aperture of 0.22

The alignment process contains two steps.

1) The facility laser is used to align the probe center to be on axis with the facility center line.

2) An alignment tool, which is centered on axis through the facility laser and contains 4 LEDs of different colors at the edges of the required view angle, is used. If the spectral signature of all 4 LEDs is visible in the spectrometer, and if the distance of the alignment tool is chosen correctly, the probe is considered to be aligned.

This alignment procedure was successful in yielding symmetric profiles during the probe sweep. Figure 8 shows a picture of the LED alignment tool during test preparation in the IHF. In lab tests, the pointing accuracy was determined by placing the LED tool - being aligned with the probe axis - at a defined distance of 9inches $(\sim 229 \mathrm{~mm})$ from the probe. The LED tool was displaced in vertical and horizontal direction by a defined amount and the resulting spectra were measured. A displacement of $1.5 \mathrm{~mm}$ in any direction (corresponding to a misalignment angle of $\sim 0.4^{\circ}$ ) yielded clearly recognizable changes in the measured spectra as shown in Fig. 9. During alignment in the facility, the default spectrum was reproduced with clearly lower deviations than obtained in these pre-test spectra, so the alignment during the tests is considered better than $\pm 0.4^{\circ}$.

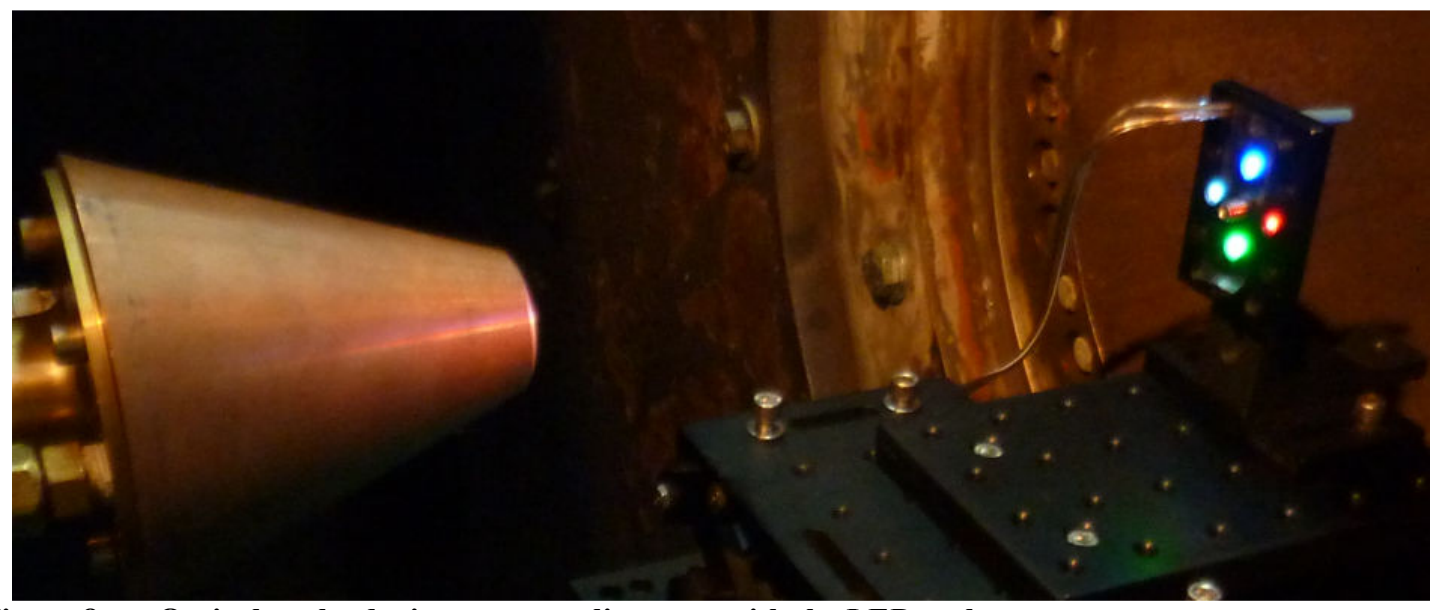

Figure 8. Optical probe during pre-test alignment with the LED tool.
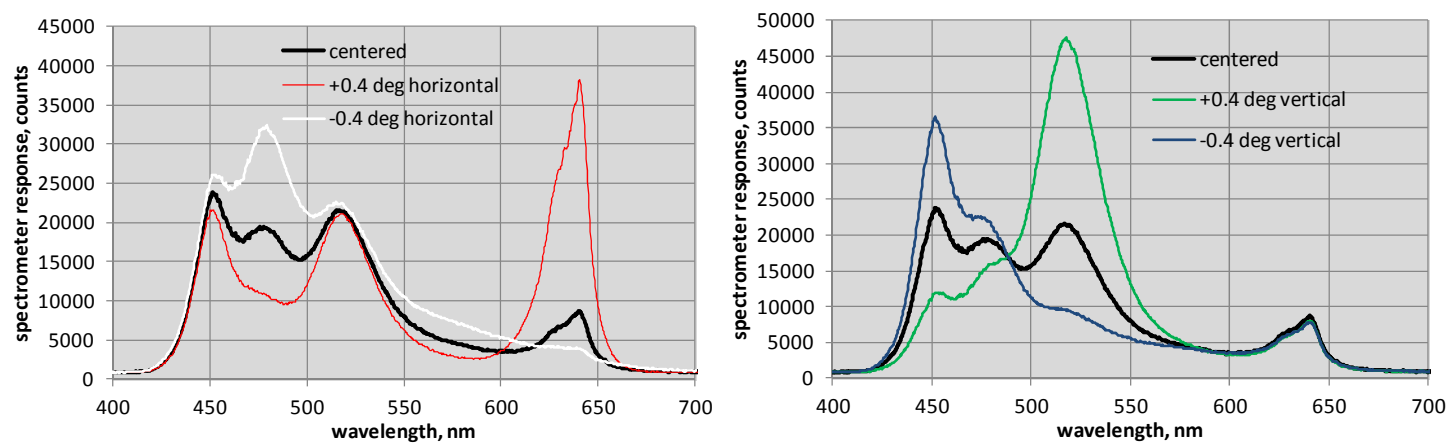

Figure 9. Spectral characteristics of the LED tool in nominal position (black) and misaligned by $0.4 \mathrm{deg}$ in each direction. The line color indicates the LED color of the dominant LED. 


\section{Calibration}

The calibration to spectral irradiance was done before and after each test using an integrating sphere with a distance to the probe surface of 9 inches $(\sim 229 \mathrm{~mm})$ which was masked down to a defined aperture of $12.65 \mathrm{~mm}$ diameter, yielding a half opening angle of about $1.6^{\circ}$, which is well within the acceptance angle of the fiber and representative for the field of view defined by the IHF nozzle throat. The spectral radiant intensity emitted by the lamp is given as the emitted radiance multiplied with the emitting area. The receiving area $A_{O F}$ (and therefore the solid angle from the lamp to the probe) is defined by the fiber diameter, the emitting area is defined by the mask in front of the integrating sphere. The incident spectral irradiance $I$ can be computed from the calibrated spectral radiance $L$ using equation (5)

$$
I(\lambda)=\frac{L(\lambda) A_{\text {emiting }} \Omega}{A_{O F}}=\frac{L(\lambda) A_{\text {emitting }}}{x_{0}{ }^{2}}
$$

$x_{0}$ being the distance from the integrating sphere exit to the optical fiber entrance inside the probe body.

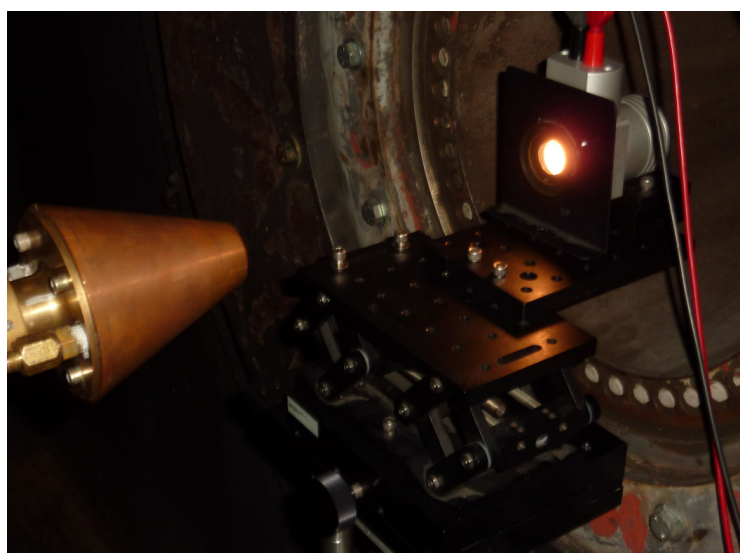

Figure 10. Calibration lamp mounted in front of the optical probe.
To increase signal to noise in the UV where the integrating sphere emission is weak, a cross calibration with a Deuterium lamp was done after completion of the test series. Although the Deuterium lamp is calibrated to spectral radiance, the magnitude depends strongly on the diameter of the measured spot. The factory calibration sheet only provides values up to $1 \mathrm{~mm}$ measurement spot size, therefore the UV calibration factor was scaled to the one obtained from the calibration with the integrating sphere in the overlapping wavelength regions of the two lamps. Hence, a calibration down to $200 \mathrm{~nm}$ could be achieved. However, the combination of optical fibers and spectrometer lost its sensitivity at about $225 \mathrm{~nm}$.

Since the arc-jet radiation was about 4-5 orders of magnitude higher than the light provided by the calibration lamps, significantly different acquisition times were used for arc-jet measurements (less than $1 \mathrm{~ms}$ ) and calibration (30 s). During lab testing, it became evident that the spectrometer showed a significant nonlinearity with acquisition time with strong gradients for times less than $3.7 \mathrm{~ms}$. Therefore, different measurements series with a variation of acquisition time while measuring the same radiation source were performed with three calibration lamps of different power. It turned out that besides the time non-linearity the detector showed a slight decrease in sensitivity once the signal approached the saturation limit. Both nonlinearities could be separated. The resulting sensitivity curves are shown in Fig. 11.
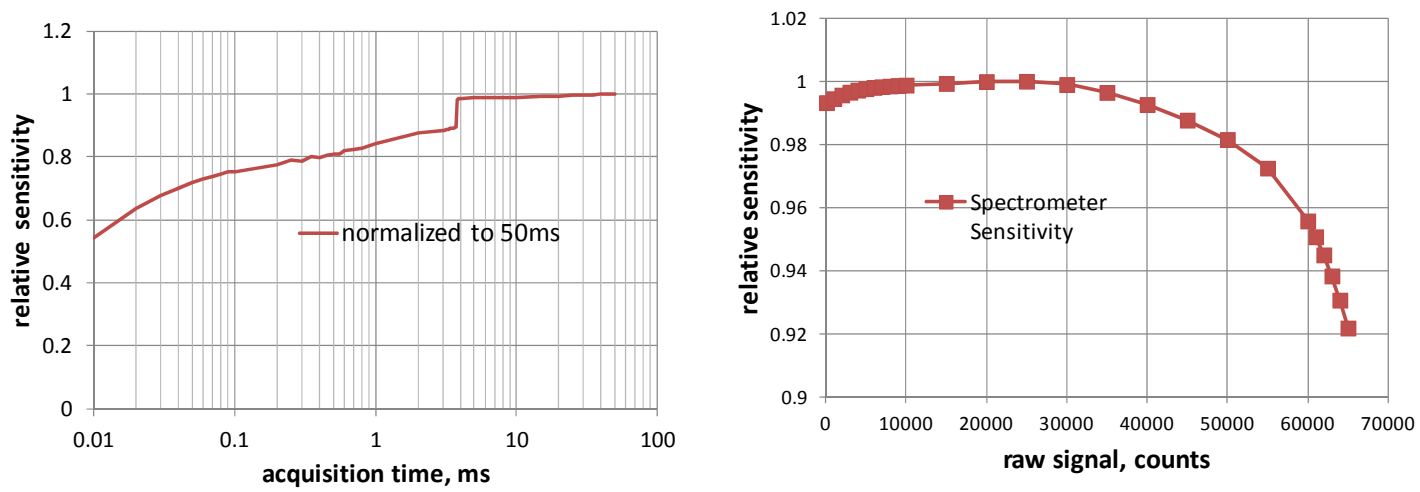

Figure 11. Relative spectrometer sensitivity vs. acquisition time (normalized to 50ms) and raw signal. 


\section{Test Conditions}

For these tests, two conditions at bulk enthalpy levels of 12 and $22 \mathrm{MJ} / \mathrm{kg}$ were chosen. These conditions, labeled as MSL-A2 and MSL-8, were chosen because there is a sizeable database of arc jet data at these conditions and computed flow field solutions are readily available. Table 1 summarizes the heater settings for the two conditions.

Table 1: Facility Settings

\begin{tabular}{|c|c|c|c|c|c|c|c|}
\hline Condition & $\begin{array}{c}\text { Arc } \\
\text { Current } \\
\text { (A) }\end{array}$ & $\begin{array}{c}\text { Arc } \\
\text { Voltage } \\
\text { (V) }\end{array}$ & $\begin{array}{c}\text { Main-Air } \\
\text { Mass Flow } \\
\text { Rate (g/sec) }\end{array}$ & $\begin{array}{c}\text { Add-Air } \\
\text { Mass Flow } \\
\text { Rate (g/sec) }\end{array}$ & $\begin{array}{c}\text { Argon } \\
\text { Mass Flow } \\
\text { Rate (g/sec) }\end{array}$ & $\begin{array}{c}\text { Chamber } \\
\text { Pressure } \\
\text { (kPa) }\end{array}$ & $\begin{array}{c}\text { Bulk } \\
\text { Enthalpy } \\
\text { (MJ/kg) }\end{array}$ \\
\hline$M S L-A 2$ & 3500 & 4330 & 270 & 330 & 42 & 486 & 12 \\
\hline$M S L-8$ & 6000 & 7300 & 740 & 55 & 54 & 886 & 22 \\
\hline
\end{tabular}

The conditions of Table 1 were run with both 6-inch and 13-inch nozzles at corresponding probe distances to the nozzle exit plane of 3 inches and 10 inches, respectively. At Ames, test stream centerline conditions are routinely measured via copper slug calorimeter probes. ${ }^{7}$ The standard probe geometries are hemispherical (hemi), uniform heat flux (iso-q), and flat-faced. The measured heat fluxes depend on calorimeter shape and material properties. The heat flux on a flat-faced or an iso-q model (these two shapes are preferably used for material testing) is substantially lower than that to a hemispherical model. Table 2 shows the measured heat flux values for the various geometries at a location of 3 inches from the exit plane of the 6-inch nozzle and 10 inches from the exit plane of the 13 inch nozzle. Where measured values with a flat-faced calorimeter were not available, calculated values based on the relation given by Zoby and Sullivan ${ }^{8}$ are shown.

Table 2: Measured centerline heat fluxes ("computed according to Zoby and Sullivan ${ }^{8}$ ):

\begin{tabular}{|c|c|c|c|c|c|}
\hline \multirow[t]{2}{*}{ Condition } & \multicolumn{2}{|c|}{$\begin{array}{c}\text { Heat flux on a } \\
102 \mathrm{~mm} \text { hemi } \\
\text { calorimeter, W/cm }\end{array}$} & \multirow{2}{*}{$\begin{array}{l}\text { Heat flux on a } \\
102 \mathrm{~mm} \text { iso-q } \\
\text { calorimeter, } \\
W / \mathrm{cm}^{2}\end{array}$} & \multirow{2}{*}{$\begin{array}{l}\text { Heat flux on } \\
\text { a flat face } \\
\text { calorimeter, } \\
W / \mathrm{cm}^{2}\end{array}$} & \multirow[t]{2}{*}{$\begin{array}{l}\text { Heat flux on } \\
\text { a sphere/cone } \\
\text { calorimeter, } \\
W / \mathrm{cm}^{2}\end{array}$} \\
\hline & Slug & Null-Point & & & \\
\hline $\begin{array}{c}\text { Cond. } 1 \text { (MSL-A2) } \\
\text { 6-inch nozzle, } 3 \text { inch location } \\
\text { 13-inch nozzle, } 10 \text { inch location }\end{array}$ & $\begin{array}{c}1036 \\
391 \\
\end{array}$ & 352 & $\begin{array}{l}928 \\
292 \\
\end{array}$ & $\begin{array}{l}581^{*} \\
219^{*}\end{array}$ & $1054(438)$ \\
\hline $\begin{array}{c}\text { Cond. } 4 \text { (MSL-8) } \\
\text { 6-inch nozzle, } 3 \text { inch location } \\
\text { 13-inch nozzle, } 10 \text { inch location }\end{array}$ & $\begin{array}{c}2013 \\
896 \\
\end{array}$ & 862 & $\begin{array}{c}1953 \\
694 \\
\end{array}$ & $\begin{array}{c}1142 * \\
648\end{array}$ & $2332(968)$ \\
\hline
\end{tabular}

Profiles of heat flux and pressure across the test stream were also obtained with the ARC trident. The trident allows three instruments to be swept across the test stream. The three instruments used for this test campaign were: a 102-mm-diameter hemispherical probe with a null point calorimeter and a pressure port; a $4.57 \mathrm{~mm}$ nose radius sphere/cone probe with a null point calorimeter; and a $4.57 \mathrm{~mm}$ nose radius sphere cone probe with a pressure port. Heating rate and pressure as a function of distance from the center of the jet for the respective probes are shown in Figs. 12 and 13 for the two test conditions. The probes were swept across the free jet at a speed of $60 \mathrm{in} / \mathrm{sec}$ (about $1.5 \mathrm{~m} / \mathrm{s}$ ). The heat transfer on the sphere/cone probe was converted to an equivalent heat transfer to a $102 \mathrm{~mm}$ hemisphere by multiplying by $\sqrt{R_{\text {SphereCone }} / R_{\text {Hemi }}}$ using an equivalent radius correlation $\left(r_{\text {eff }}=8.76 \mathrm{~mm}[0.345 \mathrm{in}]\right) .^{5}$ The centerline values of heat flux are noted in Table 2. Parenthetical numbers in Table 2 represent a normalization of the heat flux to a hemispherical geometry. 


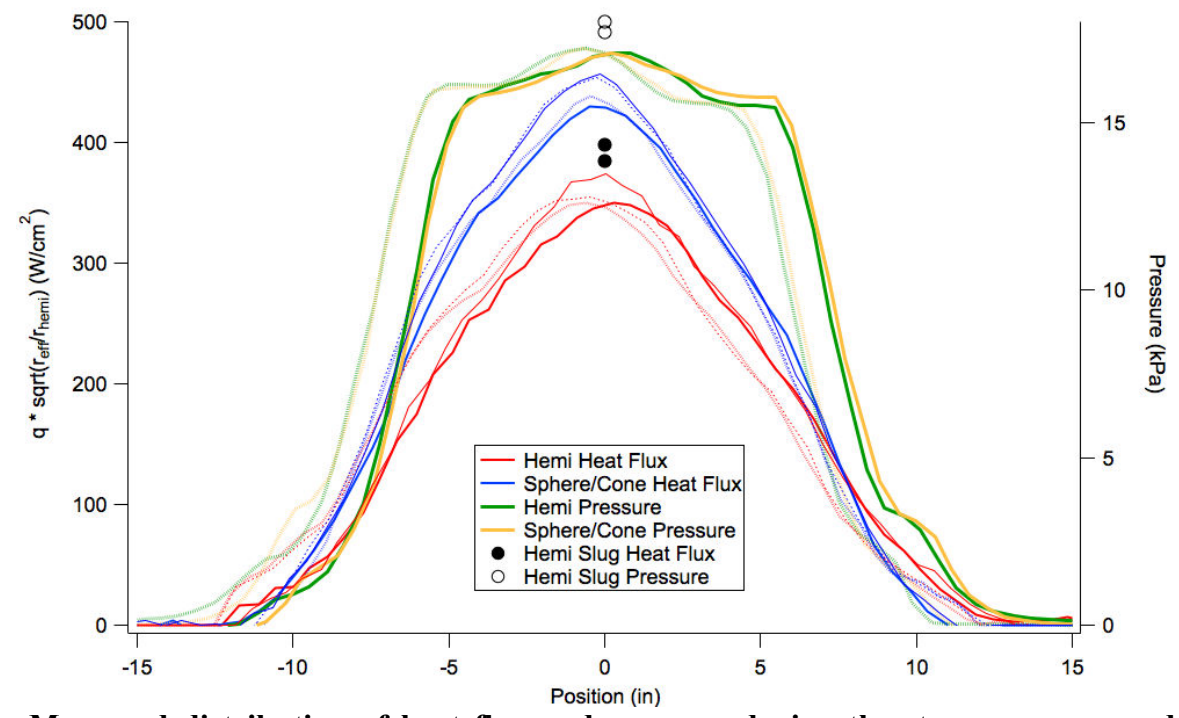

Figure 12. Measured distribution of heat flux and pressure during the stream sweeps and centerline measurements for condition MSL-A2 in the 13-inch nozzle. Multiple lines of the same color represent repeated forward and backward sweeps. Sphere/cone data have been normalized to a hemispherical geometry.

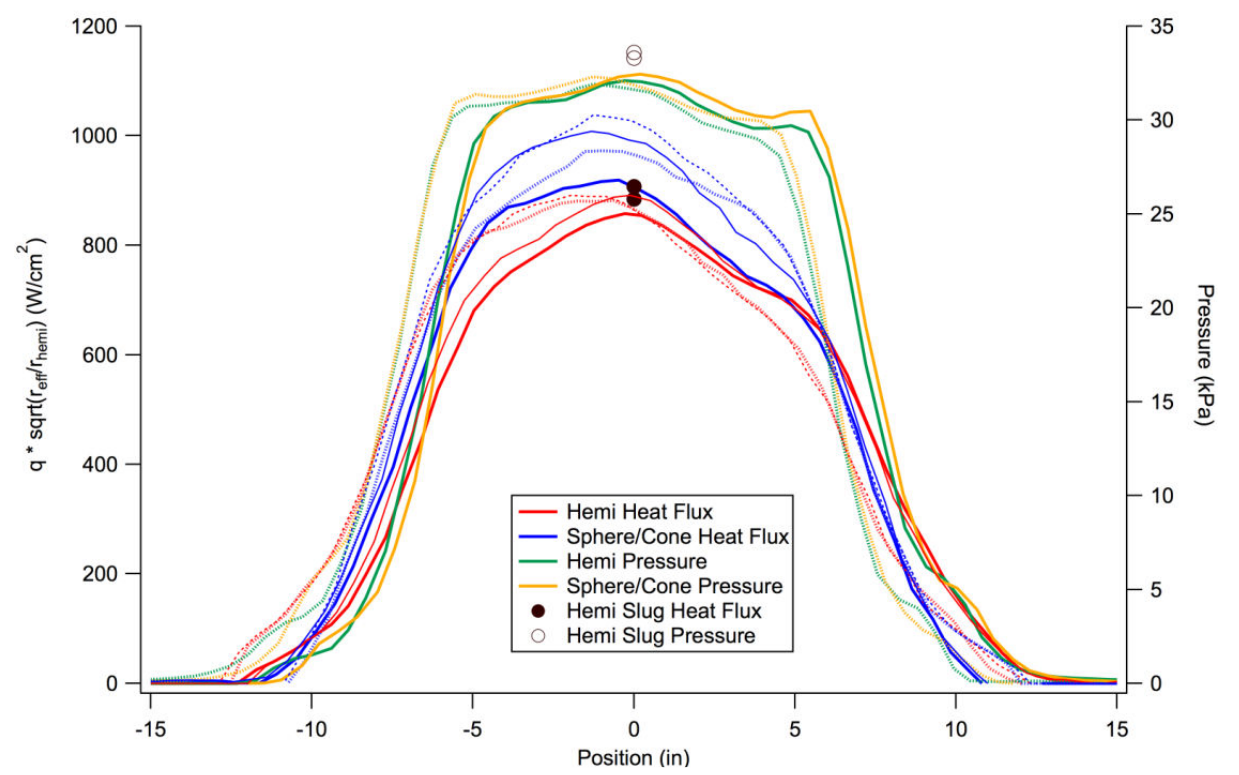

Figure 13. Measured distribution of heat flux and pressure during the stream sweeps and centerline measurements for condition MSL-8 in the 13-inch nozzle. Multiple lines of the same color represent repeated forward and backward sweeps. Sphere/cone data have been normalized to a hemispherical geometry.

\section{Theory/Modeling}

The gains made in modeling of high-temperature thermochemical nonequilibrium flowfields, and advances made in rapid and efficient numerical solvers for such models, have enabled the development of frameworks for analysis of arc-heated flows expanded using a nozzle. ${ }^{5}$ The analysis framework makes use of the fact that the geometry of test nozzles is well known, and that the nozzle-calorimeter combinations are usually fixed. Therefore, there is only a one-time investment in building grids required for numerical simulation - grid generation is usually a long-lead item in the computational process. The computational domain contains the plenum, the convergent-divergent nozzle, and the free jet. Since the nozzles are almost always underexpanded (i.e., $p_{\text {exit }}>p_{\text {test chamber }}$ ), the free jet is truncated in such a way as to allow imposition of supersonic extrapolation at the boundaries. The calorimeter/test article is considered as an integral part of the computational domain. Such an approach allows for consideration of flow nonuniformities (in enthalpy and/or mass flux). In order to 
speed up the analysis process, axial symmetry is assumed, thus reducing the problem from three dimensions to two. Although the analysis process has been streamlined, there are several uncertainties and unanswered questions regarding the flow, especially the radial distribution of enthalpy (i.e., enthalpy non-uniformity) in the jet. Figure 14 shows the numerically computed enthalpy distribution in plenum and 13-inch nozzle of the IHF.

In the present effort, a volume grid has been constructed for the 13-inch and 6-inch nozzles of the IHF and free jet without any calorimeter or test article. The axisymmetric version of the CFD code DPLR $R^{3}$ solves the equations governing the temporal development (to steady state) of a flow consisting of 12-species $\left(\mathrm{N}_{2}, \mathrm{O}_{2}, \mathrm{NO}_{\text {, }}\right.$ $\mathrm{NO}^{+}, \mathrm{N}_{2}{ }^{+}, \mathrm{O}_{2}{ }^{+}, \mathrm{N}, \mathrm{O}, \mathrm{N}^{+}, \mathrm{O}^{+}, \mathrm{e}^{-}, \mathrm{Ar}$ ) in thermochemical nonequilibrium. The facility measured flow rates (of air and argon), and arc chamber pressure are used to estimate the bulk enthalpy of the arc-heated flow in the plenum section. Using an in-house code, NOZZLE_THROAT_CONDITIONS, flow non-uniformities in enthalpy and mass flux are considered (Gaussian distributions are considered based on nullpoint surveys of the free jet). Further details of the computational process/procedure can be found in Ref. 5.

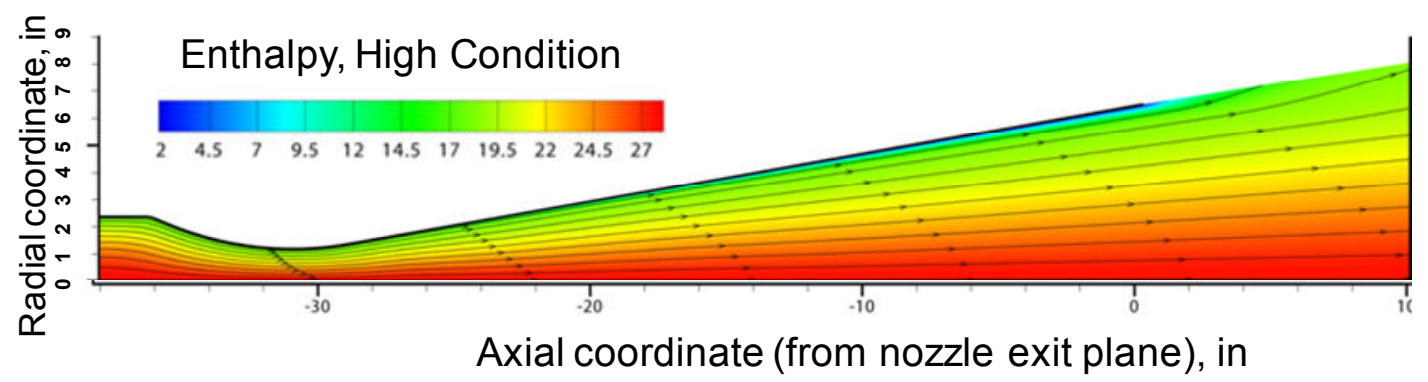

Figure 14. Numerically computed enthalpy distribution in plenum and 13-inch nozzle of the IHF.

From the converged solution, profiles of the translational/rotational and vibro-electronic temperatures, and number densities of all the constituent species are extracted at a line of sight perpendicular to the flow through the center of the plenum region and along a line of sight on the heater axis from the plenum through the nozzle. Figure 15 illustrates the radial profiles of particle densities (in parts $/ \mathrm{m}^{3}$ ) and temperatures in the plenum for the MSL-8 as obtained from the CFD solution.

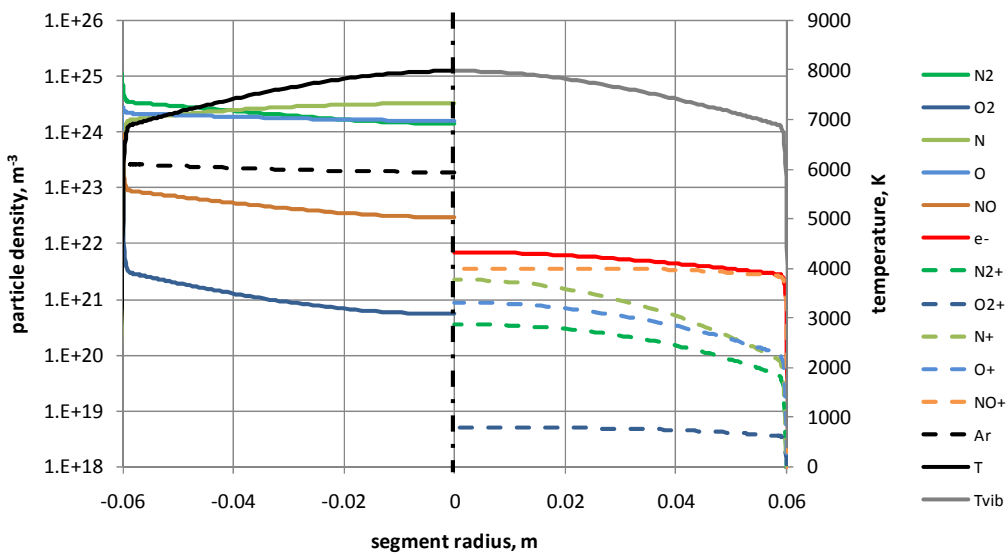

Figure 15. Simulated temperatures and particle densities (neutrals left, ions right) along the centerline in the IHF plenum region for the MSL-8 condition.

This line of sight is equivalent to the center line measurement during the plenum emission spectroscopy described in detail in Ref. 1 . Temperatures between $7000 \mathrm{~K}$ and $8000 \mathrm{~K}$ with a rather flat profile are obtained. Oxygen $\left(\mathrm{O}_{2}\right)$ is almost fully dissociated over the whole cross section, nitrogen dissociation reaches about $70 \%$ at the center, and ionization reaches a maximum of roughly $0.1 \%$, with the main ion being $\mathrm{NO}^{+}$.

For the optical probe field of view, NEQAIR simulations were performed along lines of sight, starting in the plenum and ending in the free stream at 10 inches distance to the probe, based on the thermochemical data from $D P L R$ simulations for the high enthalpy condition. The axial profiles of temperatures and particle densities obtained from DPLR are shown in Fig. 16. For an initial discretization of this profile, three lines of sight from the plenum to probe position were used, defined by offsets of $0 \mathrm{~mm}$ (center line), $15 \mathrm{~mm}$ and $25 \mathrm{~mm}$ in the nozzle throat. The corresponding plasma states were considered constant over the corresponding ring cross sections at the different axial positions. For more detailed investigation, the discretization will be refined in future investigations. The ratio of these ring areas does not change in the detected cone shape volume, so the resulting spectrum can be derived from weighing the spectra by the ratio of these ring areas $(0.56 / 0.33 / 0.11$ for the outer/intermediate/inner ring areas). Fig. 17 illustrates the geometrical situation for the simulation of lines of sight in the field of view of the optical probe. For the sake of visibility, the vertical scaling of the IHF geometry was enlarged by a factor of two. 


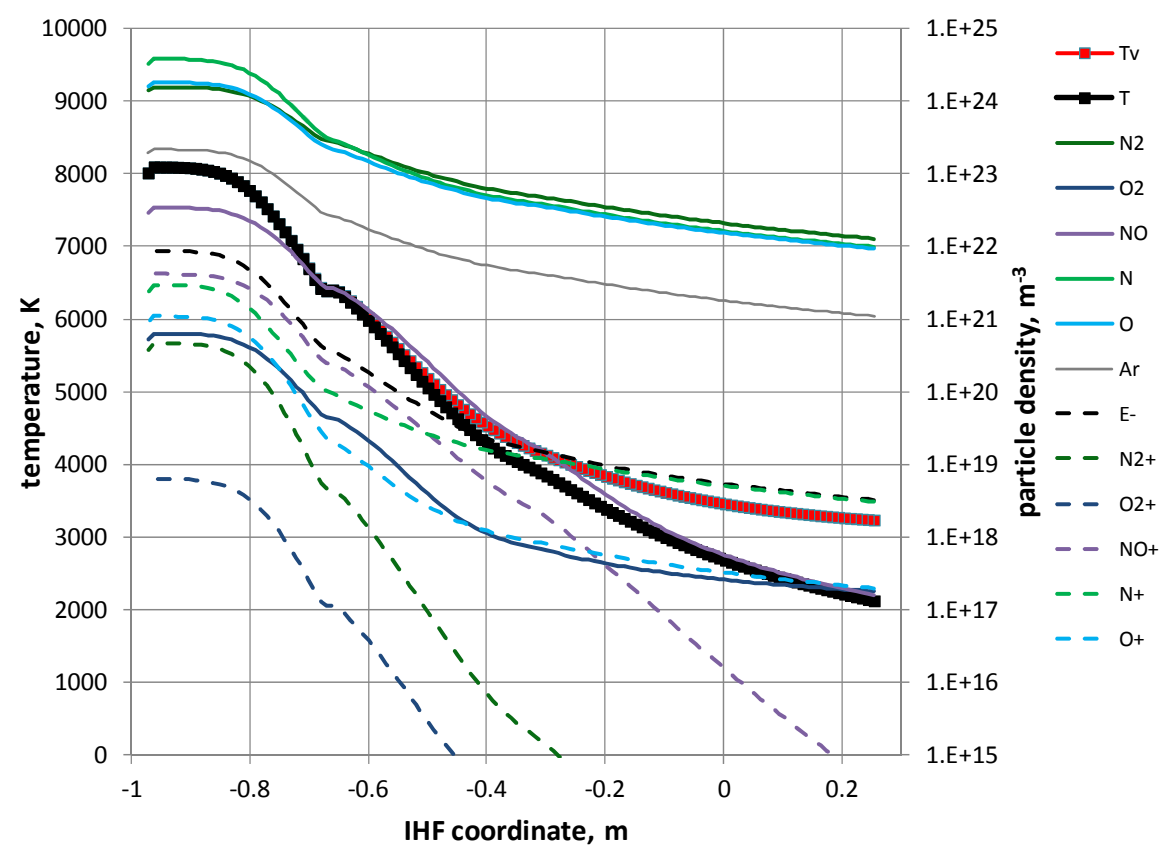

Figure 16. Simulated temperatures and particle densities along the axial centerline from the plenum section to the probe position (probe was not modeled) for the high enthalpy condition in the 13-inch nozzle. The nozzle exit is chosen as 0 , therefore positions inside the heater are at negative values.

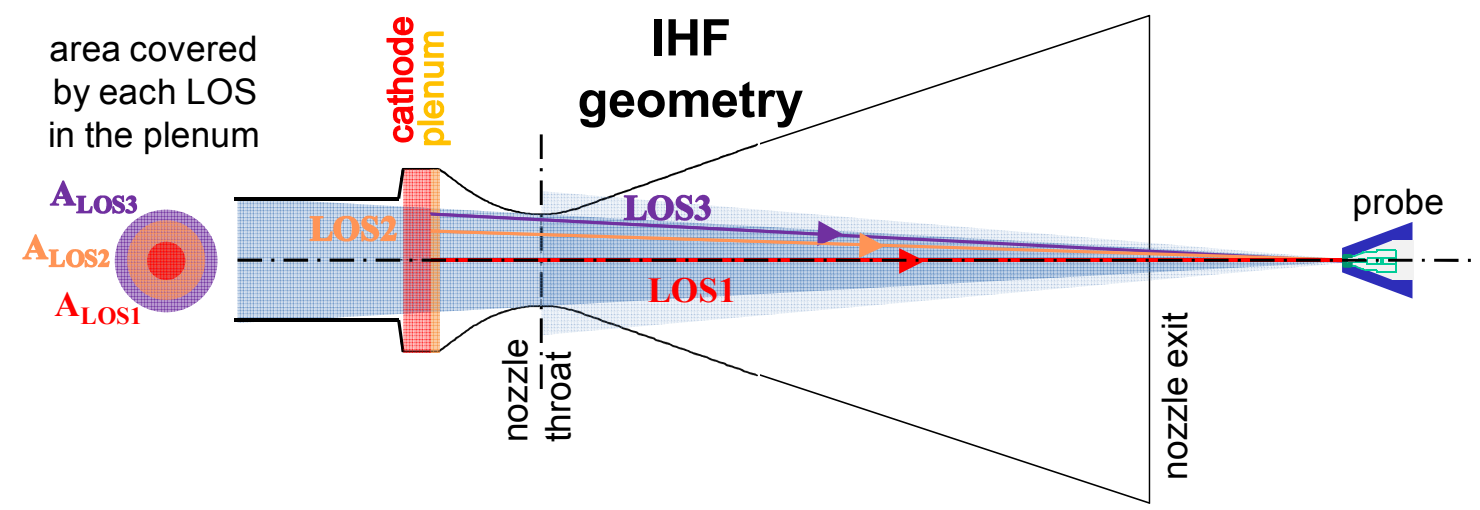

Figure 17. Illustration of the field of view of the optical probe (maximum: light blue, effective: darker blue) inside the IHF heater with lines of sight (LOSs: colored arrows) used for the NEQAIR computations and effective areas covered by these lines of sight in the plenum region.

The extracted profiles are considered to be the simulated line of sight, and the data are used in the code NEQAIR $2009 \mathrm{~V} 6^{6}$ which is a line-by-line spectroscopic code with one-dimensional transport of radiative intensity. It computes the emission and absorption of atoms $(\mathrm{N}, \mathrm{O}, \mathrm{H}, \mathrm{C}, \mathrm{He})$ and molecules $\left(\mathrm{N}_{2}, \mathrm{~N}_{2}{ }^{+}, \mathrm{NO}, \mathrm{O}_{2}\right.$, $\mathrm{H}_{2}, \mathrm{CO}, \mathrm{C}_{2}, \mathrm{CN}$ ) as well as bound-free and free-free continuum radiation caused by interactions of electrons with neutral and ionized atomic species. Line broadening due to Doppler, Stark, resonance, and Van der Waals mechanisms as well as the natural line width realized through Voigt line shapes are included, and an additional Voigt broadening can be applied to account for instrument broadening. Radiative transport is computed along a line-of-sight as a series of one-dimensional cells - a tangent slab approximation. In absence of an individual electron temperature in the two temperature model used in DPLR, the vibrational temperature is used for electronic excitation.

Principal molecular band systems considered in this work are the $1^{\text {st }}$ and $2^{\text {nd }}$ positive systems of $\mathrm{N}_{2}$, the $1^{\text {st }}$ negative system of $\mathrm{N}_{2}{ }^{+}$, and the $\gamma, \beta, \delta$, and $\varepsilon$ systems of NO. High-resolution spectra were computed using NEQAIR for these band systems and were convolved with the experimentally determined instrument function to produce synthetic spectra for comparison against the measurements. 
For each distance to the probe, the view factor depends on the radiating area $A_{e m}$ and the solid angle to the receiving surface. Using equation (5) the spectral incident irradiance to the probe $I_{\text {probe }}$ can be written as:

$$
I_{\text {probe }}=\frac{L_{\text {plasma }} A_{e m}}{x_{0}{ }^{2}}
$$

In the conical part of the field of view (i.e. until the effective area gets larger than the radiating area defined by the heater contour), this view factor is a constant since the effective area grows with the sine of the distance squared and the solid angle decreases with the square of the distance.

$$
I_{\text {probe, cone }}=\frac{L_{\text {plasma }} \pi\left(x_{0} \tan (\alpha / 2)\right)^{2}}{x_{0}{ }^{2}}=L_{\text {plasma }} \pi\left(r_{N T} / x_{N T}\right)^{2}=\text { const } \text {. }
$$

with the opening angle of the effective field of view $\alpha$, the distance to the nozzle throat $x_{N T}$, the nozzle throat radius $r_{N T}$, and the distance to the probe $x_{0}$.

To determine the contributions of plasma emission in the different wavelength ranges, the NEQAIR computations were performed in the wavelength region from $70 \mathrm{~nm}$ to $3.2 \mu \mathrm{m}$. Figure 18 shows the combined spectral irradiance at the probe position as a weighted average of the three lines of sight under consideration on linear and logarithmic scale. The major contribution comes from the wavelength region from the UV to the NIR, the vacuum UV contributes with about $36 \%$, the infrared only with about $6 \%$. The contributions of the different wavelength regions are summarized in Fig. 19.

To check for the contributions of the different regions of the heater, separate computations were done for the plenum, the converging nozzle section, the diverging nozzle, and the free stream. The contributions vary slightly with wavelength due to different absorption with wavelength but, as expected, the free stream region does not contribute significantly. The contribution of the divergent nozzle region, although representing the largest volume, emits less than $10 \%$ of the total radiation as shown in Fig 20.

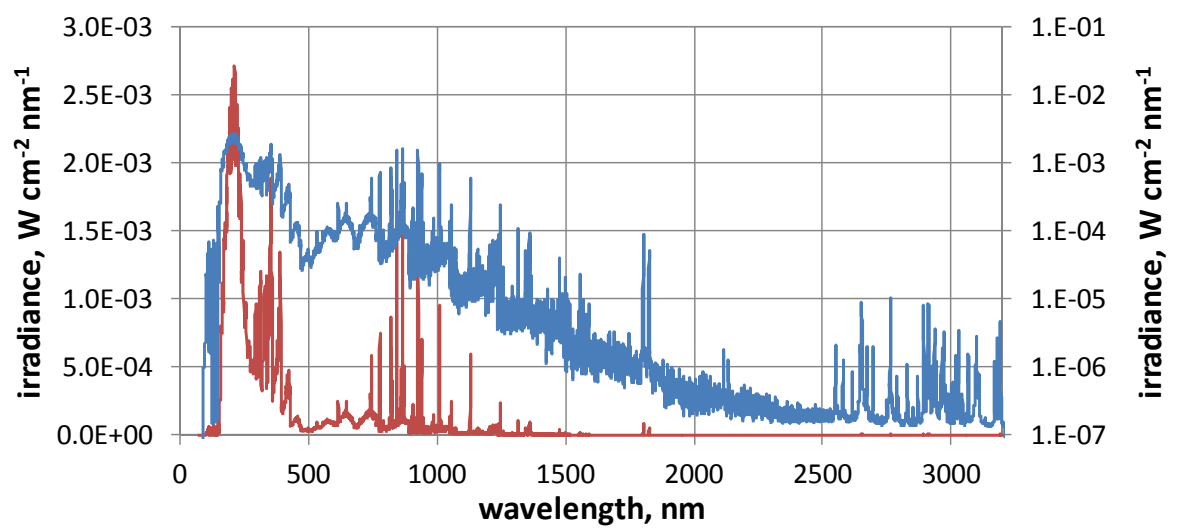

Figure 18. Area averaged, simulated spectral irradiance incident at the probe position from $N E Q A I R$ computations based on DPLR simulation on linear (red) and log scale (blue).
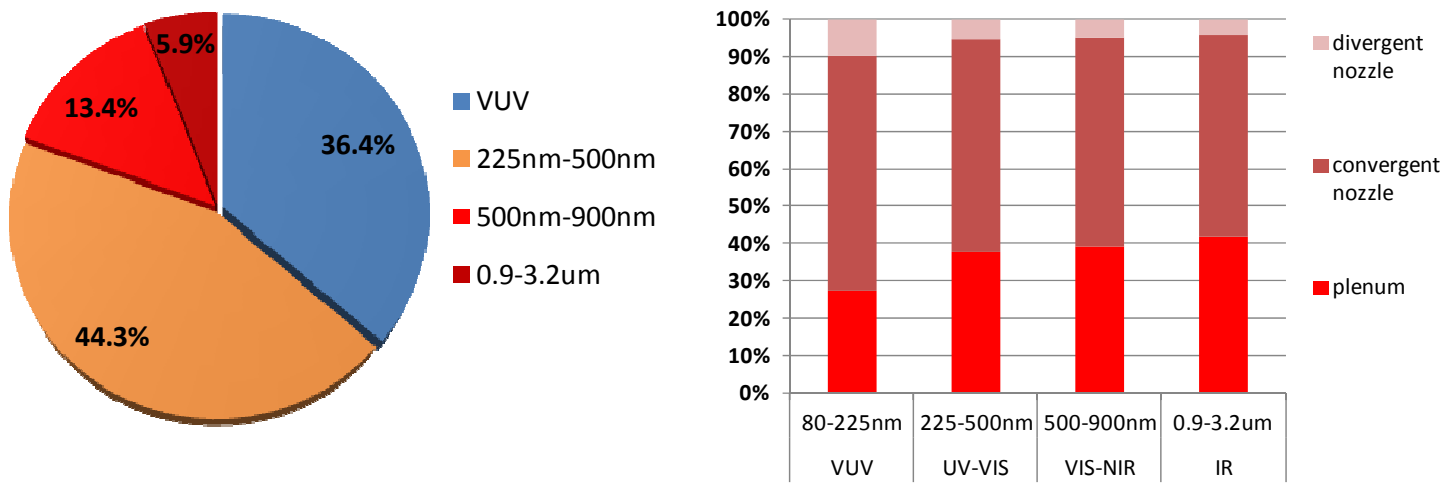

Figure 19. Contributions of the different Figure 20. Contributions of the different heater wavelength regions to the total incident regions to the incident radiation at the probe position radiation at the probe position from NEQAIR in different wavelength regions from NEQAIR computations. 


\section{Measured Spectra and Radiation Heat Fluxes}

In Fig. 21 the spectral distribution of incident radiation between 225 and 900nm on the IHF center-line, calibrated to incident irradiance (or spectral radiative heat flux) to the probe surface is shown. The radiation heat flux in this wavelength range was determined by summing the emission spectra and multiplying with the corresponding wavelength increment for each pixel yielding radiative heat fluxes of 3.3 and $8.4 \mathrm{~W} / \mathrm{cm}^{2}$ for the MSL-A2 condition (bulk enthalpy $12 \mathrm{MJ} / \mathrm{kg}$ ) and the MSL-8 condition (bulk enthalpy $22 \mathrm{MJ} / \mathrm{kg}$ ), respectively. These values were obtained with the probe positioned at 10 inches $(254 \mathrm{~mm})$ distance to the nozzle exit of the heater with the 13 inch $(330 \mathrm{~mm})$ diameter.

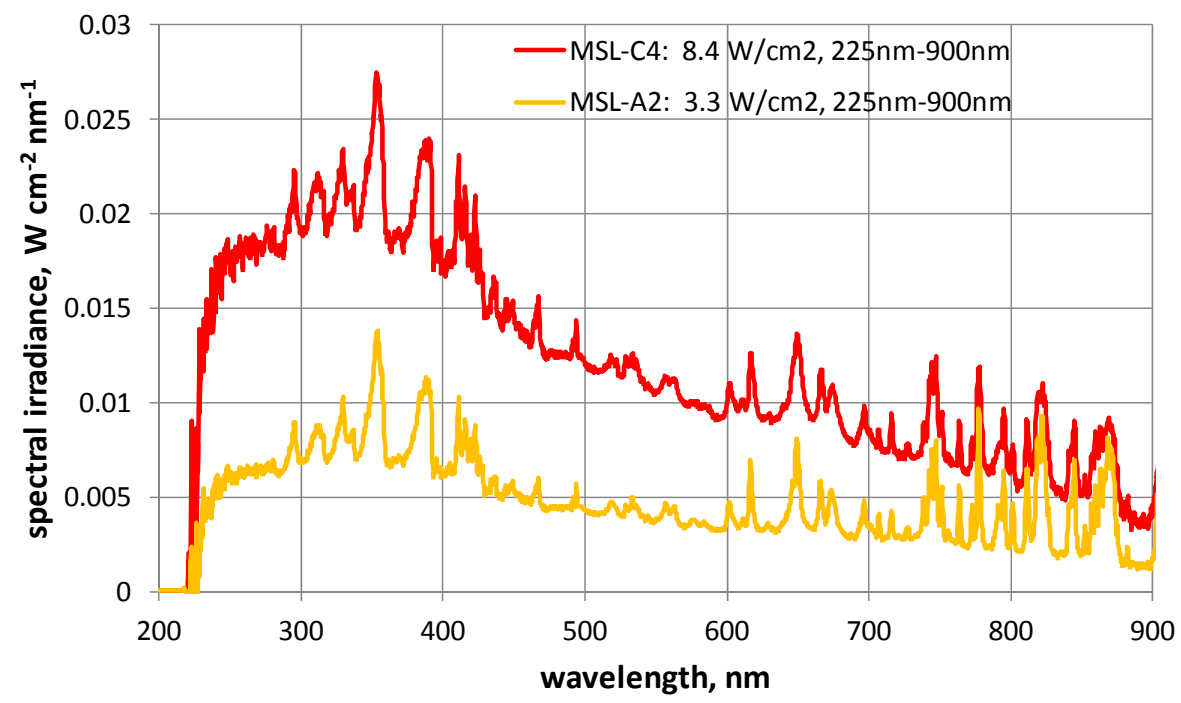

Figure 21. Measured spectral irradiance at conditions MSL-A2and MSL-8 with the IHF 13-inch nozzle in a distance of 10 inches to the nozzle exit and corresponding radiation heat flux between 225 and $900 \mathrm{~nm}$.

The main radiation at wavelengths above $600 \mathrm{~nm}$ comes from oxygen and nitrogen atom lines with a strong underlying continuum. Below 500nm, the molecular bands from $\mathrm{N}_{2}$ and $\mathrm{N}_{2}^{+}$in the near ultraviolet are strong emitters, the underlying continuum increases with lower wavelengths and peaks between 300 and $400 \mathrm{~nm}$. The total radiation load is expected to be significantly higher due to missing radiation in the vacuum UV and IR, but is still negligible in comparison to the convective heat fluxes reported in Table 2.

In parallel to the probe measurements, the plenum radiation was measured in the wavelength region from $500 \mathrm{~nm}$ to $900 \mathrm{~nm}$, similar to measurements reported previously. ${ }^{1}$ In the current test campaign, only one centerline spectrum through the plenum could be measured due to a change of the optical disk design and unavailability of optical plugs for the off-axis lines of sight. Figure 22 shows the center line perpendicular to the flow in the plenum region of the arc-heater and the corresponding radiation computed with NEQAIR based on the CFD simulation with $D P L R$. The measured spectra are higher by a factor of 2 , and the molecular radiation below $620 \mathrm{~nm}$ is significantly lower than predicted.

In the plenum, the major emission comes from atom lines and molecular bands. The continuum radiation observed with the optical probe from a downstream position is not seen in the plenum measurements. Therefore, at least the continuum portion of the radiation detected with the optical probe is anticipated to originate in the arc section upstream from the plenum, most probably due to high electron densities in the arc. Since the arc itself is not explicitly modeled in DPLR, the continuum portion of the measured spectra could not be simulated through the combination of $D P L R / N E Q A I R$ predictions. To compare the spectral irradiance measured with the optical probe to the simulation, a continuum fit to the measured data was performed as shown in Fig 23. The difference between the measured spectrum and this continuum fit (in the following referred to as "measured line spectrum") is compared to NEQAIR simulation. 


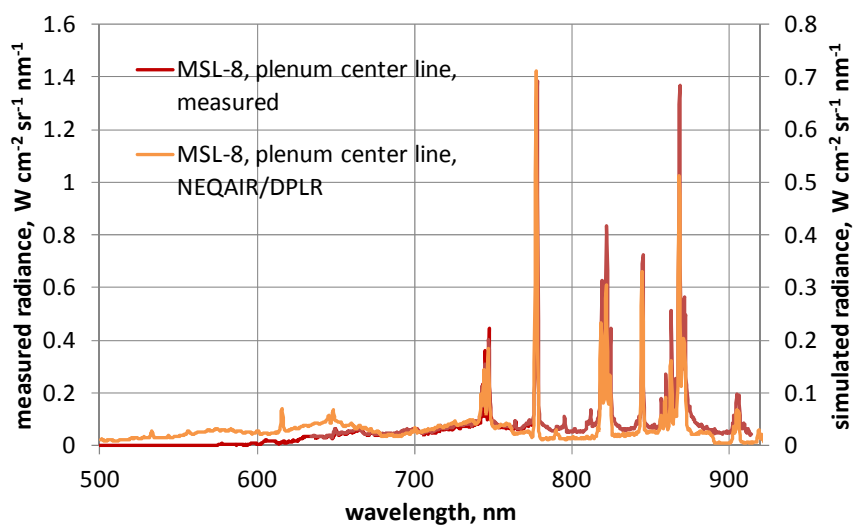

Figure 22. Measured spectral irradiance at the MSL-8 condition at the plenum center line perpendicular to the flow.

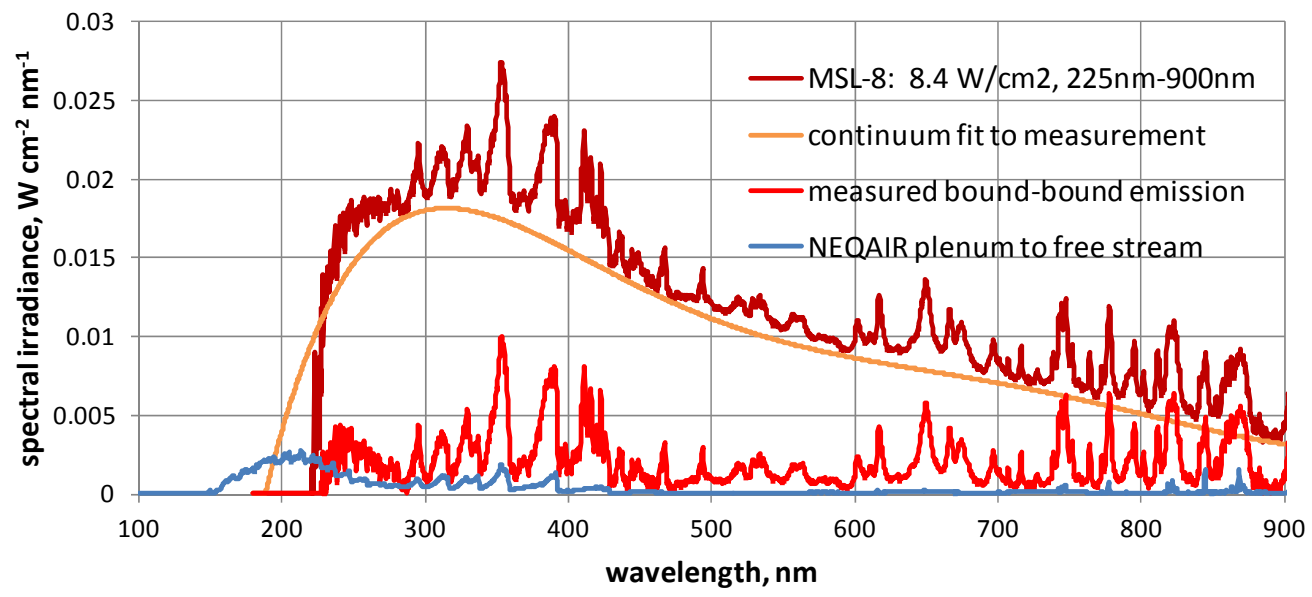

Figure 23. Measured spectral irradiance at the MSL-8 condition, continuum fit to this spectrum, resulting line spectrum, and simulated spectral irradiance from NEQAIR simulations up to the plenum region.

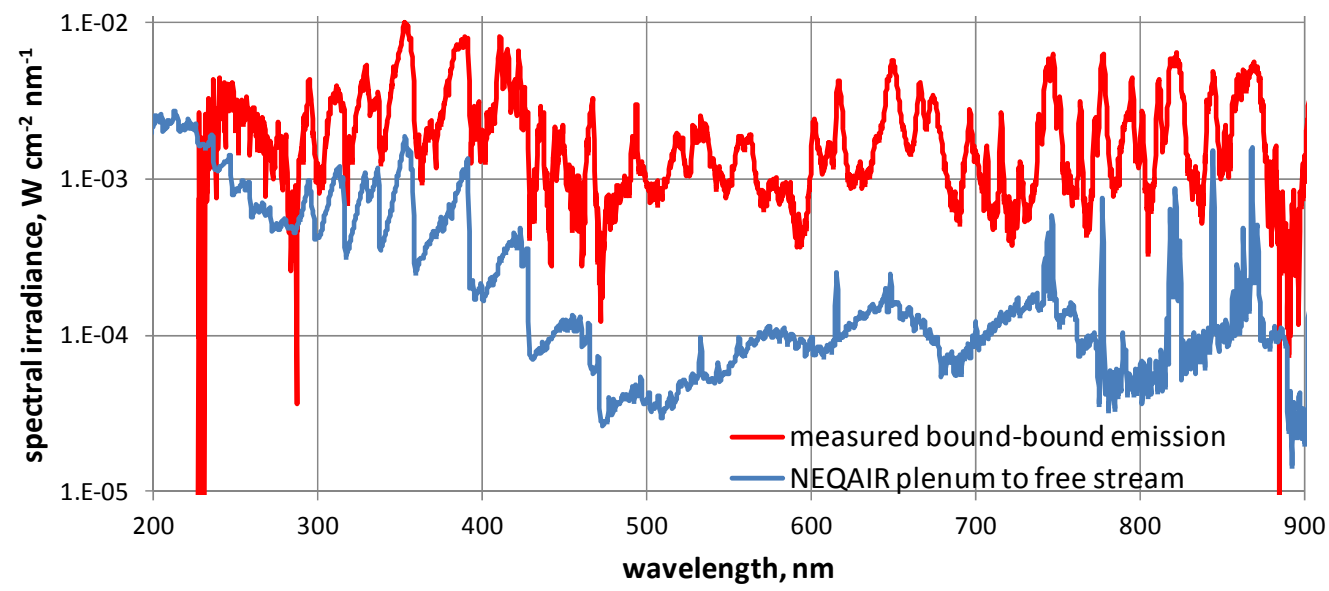

Figure 24. Measured line spectrum at the MSL-8 condition and simulated spectral irradiance from NEQAIR simulations up to the plenum region on log scale.

At wavelengths below 400nm (dominated by molecular emission) the qualitative agreement between measurement and simulation is fair. At higher wavelength, the same features (i.e., atomic lines of nitrogen and oxygen) can be recognized but the measured lines are much broader than predicted by NEQAIR. This could be 
due to Stark broadening (an effect of a high electron density in the arc section), and/or self absorption along the optical path. The different broadening, however, makes it difficult to compare the spectral irradiance directly. Therefore, the irradiance values are spectrally integrated in two different wavelength regions (UV-VIS: $225 \mathrm{~nm}$ to $500 \mathrm{~nm}$, and VIS-NIR: $500 \mathrm{~nm}$ to $900 \mathrm{~nm}$ ) for a quantitative comparison. In the UV-VIS, the simulated radiative heat flux is about $22 \%$ of the one obtained from the measured line spectrum, in the VIS-NIR, only about $6 \%$ of the measured value is reached. Of these values, about $40 \%$ are generated in the plenum region (compare Fig 20). The plenum measurements show that the VIS-NIR radiation in the plenum region is underpredicted by a factor of about two, which yields the parenthetical values in Table 3 . In conclusion, the radiation heat flux computed for the plenum and all regions downstream does account for not more than $17 \%$ of the line radiation measured by the optical probe and about $3 \%$ of the total radiation heat flux. Therefore, $97 \%$ of the measured radiation is believed to be generated in the cathode region and in the arc-column.

Table 3: Comparison of measured and simulated bound-bound radiation. Values in parens include the factor of 2 between plenum measurements and simulation (Fig. 22).

\begin{tabular}{|l|l|l|l|}
\hline Wavelength region & $\begin{array}{l}\text { Measured } \mathrm{Q}_{\mathrm{rad}} \text { from } \\
\text { line radiation }\end{array}$ & $\begin{array}{l}\text { NEQAIR } \\
\text { simulation, starting } \\
\text { in the plenum }\end{array}$ & $\begin{array}{l}\text { NEQAIR, plenum } \\
\text { only }\end{array}$ \\
\hline $225 \mathrm{~nm}-500 \mathrm{~nm}$ & 0.688 & 0.152 & 0.058 \\
\hline $500 \mathrm{~nm}-900 \mathrm{~nm}$ & 0.720 & $0.046(0.092)$ & $0.018(0.037)$ \\
\hline
\end{tabular}

The measured radiative heat flux in the wavelength region between $225 \mathrm{~nm}$ and $900 \mathrm{~nm}$ of $8.4 \mathrm{~W} / \mathrm{cm}^{2}$ is composed of about $83 \%$ continuum radiation and $17 \%$ line radiation from atoms and molecules. From the NEQAIR simulation (Fig. 20) it can be concluded that the line radiation will be increased by about $40 \%$ through radiation from the vacuum UV and the IR. Presently it is unknown how the measured continuum radiation extends into these wavelength regions, therefore these contributions are not included. Thus, a lower limit for the total radiation heat flux at the high enthalpy condition is estimated to about $9 \mathrm{~W} / \mathrm{cm}^{2}$ which corresponds to about $1.4 \%$ of the measured heat flux to a flat face calorimeter of $648 \mathrm{~W} / \mathrm{cm}^{2}$ at the MSL- 8 . This can be considered negligible for testing at the IHF with the 13 -inch nozzle at the 10inch location.

\section{Extrapolation to Testing in the 6-inch Nozzle}

An extrapolation of these results for testing with the 6-inch nozzle would be at best approximate since the radiative heat flux depends heavily on the axial distribution of emitted radiation inside the heater. However, some preliminary estimates are given here, since the 6-inch nozzle is frequently used for materials testing at high heat fluxes.

A typical test location for the 6-inch nozzle is at a distance of 3 inches to the nozzle exit. The distances to the nozzle throat with the two nozzles are 11.3 inches and 31.2 inches for the 6-inch nozzle and the 13-inch nozzle, respectively. The internal geometry of the arc-heater is the same for both nozzles. With both nozzles, the conical section of the field of view of the optical probe extends to the cathode section before the field of view is truncated by the inner wall of the heater. Therefore, the view factors for these regions are constant in both cases and a ratio of 8.3 (6-inch nozzle to 13-inch nozzle) can be calculated for the view factor. The view factors for the regions upstream from the cathode, however, approach each other with both nozzles. At a location $1.25 \mathrm{~m}$ upstream of the nozzle throat, the ratio between both nozzles decreases to 2. Figure 25 illustrates the ratio between the two view factors in the IHF internal geometry.

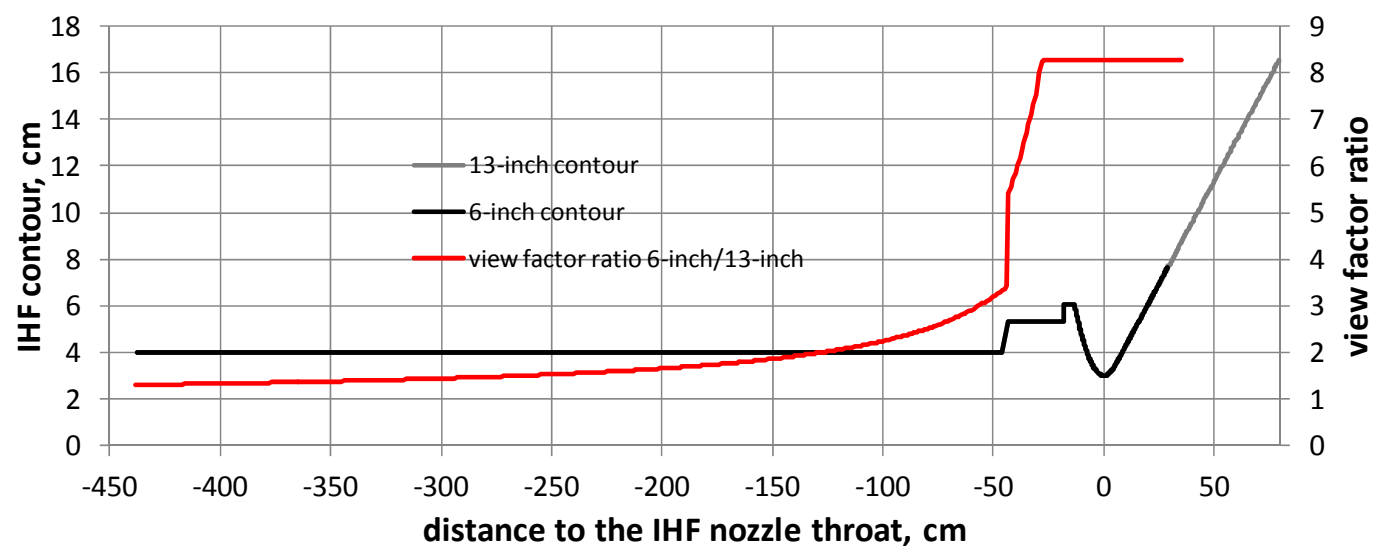

Figure 25. Ratio in view factors along the IHF axis between a test location of 10 inches distance to the 13-inch nozzle and 3 inches distance to the 6-inch nozzle for the same heater settings. 
From the comparison of measured and simulated radiation heat fluxes at 10 inches distance to the 13 -inch nozzle it can be concluded that more than $95 \%$ of the radiation heat flux is generated in the discharge region. If, as a zeroth-order approximation, the thermochemical state inside the arc is considered to be constant (i.e., the same amount of radiation is emitted at each axial position), the incident radiation can be estimated by an integration over the view factors along the heater axis. This very preliminary estimate of the contributions of the different arc-heater regions yields 30\% radiation from the cathode region and $70 \%$ from the arc column for the 13-inch nozzle configuration. With the 6-inch nozzle, this ratio would be approximately 50/50. These results would yield an estimate of an increase in radiation heat flux by a factor of 3.3 by switching to a location of 3 inches distance to the 6-inch nozzle exit. However, absorption inside the plasma is not taken into account in these considerations. If the majority of radiation is generated in the cathode region (which would be the case if the arc-region is optically thick), the factor could increase up to 8 . These radiation heating values would correspond to 3 to $7 \%$ of the heat flux to a flat face calorimeter at that position. However, a more reliable prediction requires more detailed knowledge of the axial distribution of emitted radiation. Further experiments with total radiation sensors are in preparation to measure the radiation heat fluxes in the 6-inch nozzle configuration.

\section{Summary and Conclusions}

An optical probe was designed to measure incident radiation on a sample in the arc-jet flow as part of the heat load on a material sample. A fiber-coupled spectrometer was used to measure the spectral distribution of incident radiation between 225 and $900 \mathrm{~nm}$. The radiation heat flux in this wavelength range was determined by integrating the emission spectra which were calibrated to incident irradiance. Two conditions at bulk enthalpy levels of 12 and $22 \mathrm{MJ} / \mathrm{kg}$ were investigated. With the 13-inch nozzle at a distance of 10 inches to the nozzle exit, radiative heat fluxes of 3.3 and $8.4 \mathrm{~W} / \mathrm{cm}^{2}$ were measured in the wavelength region between 225 and $900 \mathrm{~nm}$. About $17 \%$ of this radiation was caused by line radiation from atoms and molecules, $83 \%$ were continuum spectra. Based on a CFD solution for the arc-jet flow field from the plenum region to the probe position, lines of sight along the heater axis were generated and used as input for the NEQAIR radiation code. A comparison with the spectral simulation indicates that more than $95 \%$ of the measured radiation is generated in the arc region. The total radiative heat flux from the line radiation is anticipated to increase by a factor of two through contributions from wavelengths outside the measured range, i.e., from the vacuum UV and infrared. An extrapolation of the continuum radiation to these wavelength regions was not attempted. In the tested configuration, this radiation heating accounts for only about $1.4 \%$ of the nominal heat flux on a flat face model and therefore is considered negligible. From first estimates for the configuration with the 6-inch nozzle, however, the radiation heat flux is expected to increase by up to one order of magnitude and therefore might become significant. Future tests are planned with radiometers instead of a spectrometer. Thermopiles will be used as detectors due to their flat spectral response curve. To account for vacuum UV radiation, a window-less design is in preparation which will be used in parallel to a design with windows to quantify the amount of vacuum UV radiation.

\section{Acknowledgments}

The present work was supported by NASA Contract NAS2-03144 to UARC, UC Santa Cruz, and by NASA Contract NNA10DE12C to ERC Incorporated. The authors would like to thank Dr. Aga Goodsell (Chief, Reacting Flow Environments Branch, NASA ARC) for support of modeling and simulation aspects of the present work, and wish to acknowledge the support of Mark McGlaughlin, Brett Cruden, Jerry Ridge, Jay Grinstead, Lewis Ford, Vince Meglio, and the arc jet operation crew. Critical financial support was provided by NASA-SCAP for the arc-jet operational capability at NASA Ames Research Center.

\section{References}

${ }^{1}$ Winter, M W, Prabhu, D K, Taunk, J S, and Terrazas-Salinas, I, "Emission Spectroscopic Measurements in the Plenum of the NASA IHF Arc Jet Facility," AIAA-2010-4522, 10 ${ }^{\text {th }}$ AIAA/ASME Joint Thermophysics and Heat Transfer Conference, Chicago, IL, 28 Jun - 1 Jul 2010.

${ }^{2}$ Winter M W, and Prabhu, D K, "Excited State Chemistry in the Free Stream of the NASA IHF Arc Jet Facility Observed by Emission Spectroscopy," AIAA-2011-3632, 42 ${ }^{\text {nd }}$ AIAA Thermophysics Conference in Honolulu, Hawaii, 27 30 Jun 2011.

${ }^{3}$ Wright, M J, Candler, G V, and Bose, D, "Data-Parallel Line Relaxation Method of the Navier-Stokes Equations," AIAA Journal, Vol. 36, No. 9, 1998, pp. 1603-1609.

${ }^{4}$ Wright, M.W., White, T., and Mangini, N., "Data Parallel Line Relaxation (DPLR) Code User Manual Acadia Version 4.01.1," NASA/TM-2009-215388, October 2009.

${ }^{5}$ Prabhu, D K, et al., "CFD Analysis Framework for Arc-Heated Flowfields, I: Stagnation Testing in Arc-jets at NASA ARC," AIAA-2009-4080, $41^{\text {st }}$ Thermophysics Conference, June 22-25, 2009, San Antonio, TX. 
${ }^{6}$ Whiting, E E, Park, C, Liu, Y, Arnold, J O, and Paterson, J A, "NEQAIR96, Nonequilibrium and Equilibrium Radiative Transport and Spectra Program: User's Manual," NASA RP-1389, NASA, December 1996.

${ }^{7}$ Imelda, Terrazas-Salinas, J. Enrique Carballo, David M. Driver, and John Balboni, "Comparison of Heat Transfer Measurement Devices in Arc Jet Flows with Shear," AIAA 2010-5053, $10^{\text {th }}$ AIAA/ASME Joint Thermophysics and Heat Transfer Conference, 28 June - 1 July 2010, Chicago, Illinois.

${ }^{8}$ Zoby, E V and Sullivan, E M, "Effects of Corner Radius on Stagnation-Point Velocity Gradients on Blunt Axisymmetric Bodies,” NASA TMX-1067, NASA, January 1966. 